(c) ESO 2001

\title{
First XMM-Newton observations of an isolated neutron star: RX J0720.4-3125^
}

\author{
F. Paerels ${ }^{1}$, K. Mori ${ }^{1}$, C. Motch ${ }^{2}$, F. Haberl ${ }^{3}$, V. E. Zavlin ${ }^{3}$, S. Zane ${ }^{4}$, \\ G. Ramsay ${ }^{4}$, M. Cropper ${ }^{4}$, and B. Brinkman ${ }^{5}$ \\ 1 Columbia Astrophysics Laboratory, Columbia University, 550 West 120th St., New York, NY 10027, USA \\ 2 Observatoire Astronomique, CNRS UMR 7550, 11 rue de l'Université, 67000 Strasbourg, France \\ 3 Max-Planck-Institut für Extraterrestrische Physik, Giessenbachstrasse, 85748 Garching, Germany \\ ${ }^{4}$ Mullard Space Science Laboratory, University College London, Holmbury St. Mary, Dorking, \\ Surrey, RH5 6NT, UK \\ 5 SRON Laboratory for Space Research, Sorbonnelaan 2, 3584 CA Utrecht, The Netherlands
}

Received 3 October 2000 / Accepted 26 October 2000

\begin{abstract}
We present the high resolution spectrum of the isolated neutron star RX J0720.4-3125, obtained with the Reflection Grating Spectrometer on XMM-Newton, complemented with the broad band spectrum observed with the EPIC PN camera. The spectrum appears smooth, with no evidence for strong photospheric absorption or emission features. We briefly discuss the implications of our failure to detect structure in the spectrum.
\end{abstract}

Key words. stars: atmospheres - stars: individual (RX J0720.4-3125) - stars: neutron - X-rays: stars

\section{Introduction}

There is now compelling evidence that the ROSAT source RX J0720.4-3125, first discovered in a systematic survey of the galactic plane (Haberl et al. 1997), is a nearby isolated neutron star. Its spectrum appears very soft $\left(k T_{\mathrm{e}} \sim\right.$ $80 \mathrm{eV}$ ), and there is little attenuation of the X-ray flux by interstellar absorption. There is a plausible optical counterpart (Motch \& Haberl 1998; Kulkarni \& van Kerkwijk 1998), and the measured X-ray to optical flux ratio basically rules out an accreting (low-mass) X-ray binary, or an isolated white dwarf. Perhaps the strongest piece of evidence is the detection of a fairly shallow, quasi-sinusoidal modulation of the X-ray flux, at a period of $8.39 \mathrm{~s}$ (Haberl et al. 1997). The stability of this period, as measured with ROSAT over the period 1993-1996, suggests that we are seeing modulation by the spin of a compact object, most likely a neutron star.

The sinusoidal shape of the modulation in turn suggests that it is due to changing visibility, and possibly limb-darkening effects, of a hot polar cap (or polar caps), against a cooler atmosphere. Such a region could be heated by accretion from the interstellar medium, with the accretion stream focused by a magnetic field of order

Send offprint requests to: F. Paerels

* Based on observations obtained with XMM-Newton, an ESA science mission with instruments and contributions directly funded by ESA Member States and the USA (NASA).
$B_{\text {surface }} \lesssim 10^{10} \mathrm{G}$ (the field can be no stronger, or else it would prevent accretion onto the surface, given the spin period and likely values for the density of the insterstellar medium). Alternatively, the object could be releasing heat from decay of a very strong magnetic field, which would at the same time modulate the heat flow through the atmosphere due to the influence of the field on the thermal conduction in the direction transverse to the field (Greenstein \& Hartke 1983).

These issues can be directly addressed by studying both the spin history, as well as the detailed photospheric emission spectrum of the object. With XMM-Newton, we have recently obtained data of superior sensitivity that will allow us to pursue both these possibilities.

In this Letter we present a preliminary analysis of the high-resolution spectrum of RX J0720.4-3125, obtained with the Reflection Grating Spectrometer (RGS; den Herder et al. 2001), which covers the range 5-35 $\AA$ at an approximately constant resolution $\Delta \lambda=0.05 \AA$. Data were obtained simultaneously with the EPIC focal plane imaging cameras (Turner et al. 2001), of which the PN camera (Strüder et al. 2001) offers the combination of high sensitivity and high time resolution. This latter dataset will be used to investigate the evolution of the spin period. Unfortunately, however, we currently lack the satellite dynamical data to perform an accurate barycentric correction to the photon arrival times, and we defer an 
accurate measurement of the spin period to a forthcoming paper.

\section{Photospheric spectroscopy}

\subsection{Data analysis}

XMM-Newton (Jansen et al. 2001) observed our target RX J0720.4-3125 on 13 May 2000, during the Calibration/Performance Verification phase of the mission, for a total of $62.5 \mathrm{ksec}$ (continous viewing). The data were processed using the standard RGS pipeline software, provided within the SAS (Scientific Analysis System) package. Reconstructed events are placed on a common dispersion-angle and CCD pulse height scale. Bad pixels are removed from the data, and an exposure map is calculated to correct for the resulting spatial modulation of the sensitivity. Based on the known geometry of the spectrometers, dispersion angles are converted to wavelength (for details of the preflight and in-flight wavelength calibration, we refer to den Herder et al. 2001). The resulting photon list is four dimensional: each event is labeled with wavelength (or dispersion angle), crossdispersion coordinate, CCD pulse height, and CCD frame number (time). A spectrum is assembled by imposing a joint spatial/CCD-spectral filter on the data. This filter also effectively selects between spectral orders, which appear offset in dispersion angle/CCD pulse height space. Background is determined from repeating this extraction for a region on the focal plane detector parallel to the source spectrum, but offset in the cross-dispersion direction. The response of the spectrometer associated with the choice of spatial/pulse height filters can be generated automatically from an explicit model for the spectrometer (optics and detectors).

Given the 8.39 s periodicity of the source, we tried to optimize time resolution and sensitivity of the RGS, in order to perform phase-resolved spectroscopy. In principle, the RGS focal plane CCD detectors can be read out in the cross-dispersion direction in continuous clocking mode. This provides a photon arrival time resolution of $\approx 15 \mathrm{msec}$, but at the expense of the cross-dispersion imaging information. This limits the application of this High Time Resolution mode to bright sources only. On the other hand, the standard readout sequence of the nine CCD chips that make up one focal plane detector requires $5.7 \mathrm{~s}$, which is too long to allow for a meaningful phase resolution in the case of RX J0720.4-3125. We therefore decided to operate one spectrometer (RGS1) in standard imaging mode (5.7 $\mathrm{s}$ time resolution), while we chose a custom readout sequence for the other spectrometer. We interleaved a readout of CCD chip nr 3 in RGS2 with the sequential readouts of the other chips. This chip covers the approximate wavelength range $24-28 \AA$, which is therefore read out every $1.1 \mathrm{~s}$. This compromise between time resolution and spectral coverage was based on the fact that the spectrum appears as a very soft $\left(k T_{\mathrm{BB}} \approx 80 \mathrm{eV}\right)$ blackbody in the ROSAT PSPC, which we expected to

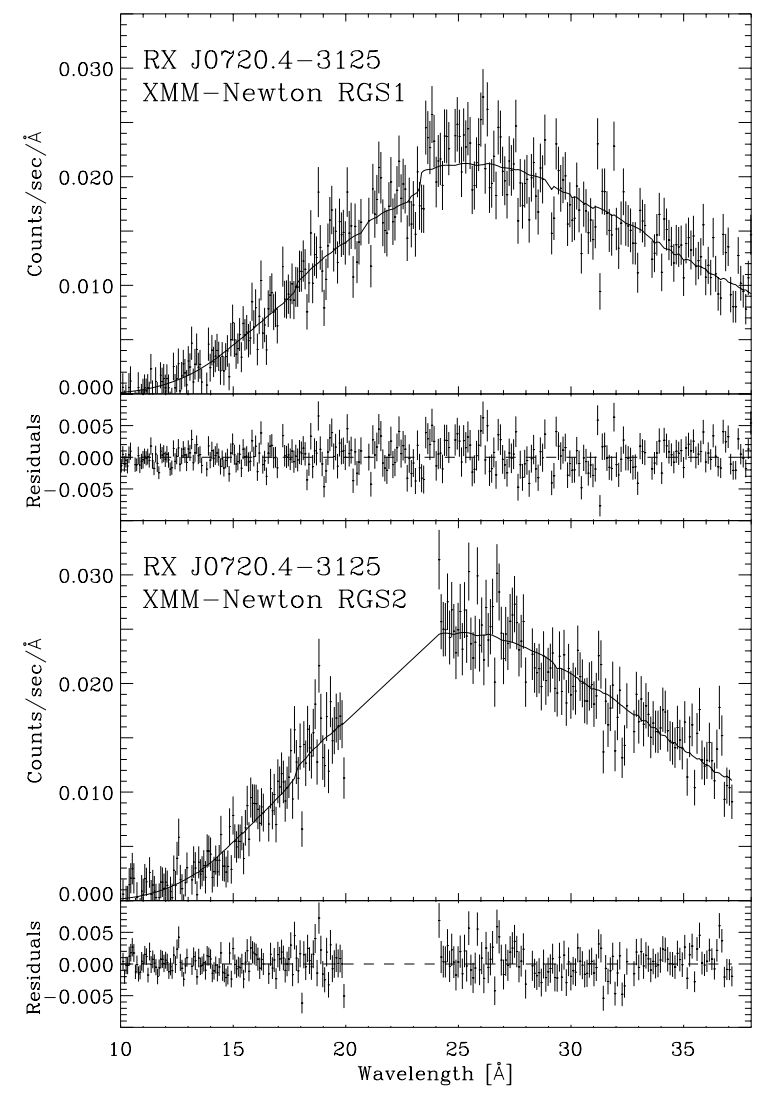

Fig. 1. The spectrum of RX J0720.4-3125, as observed with both RGS1 and 2. The data have been binned in $0.1 \AA$ bins. The range 20.1-23.9 $\AA$ is not present in the data from RGS2, because of malfunction of the drive electronics for one CCD chip in this spectrometer. Average total source count rate is 0.3 counts s $^{-1}$ in first order, per spectrometer. Superimposed is a blackbody spectral shape (see text). The slight modulation of the RGS1 post-fit residuals around $\sim 23 \AA$ is due to the presence of an $\mathrm{O} \mathrm{K}$ edge in the RGS efficiency which has not yet been fully calibrated

peak around $20 \AA$ in the RGS. In principle, therefore, we can phase resolve the 24-28 $\AA$ region of the spectrum. In this paper, however, we will restrict the discussion to the phase-averaged RGS spectrum.

\subsection{Blackbody fits}

Figure 1 shows the spectra from the two RGS's. Overlaid on the data is a simple blackbody spectrum, which, somewhat suprisingly and disappointingly, turns out to be an acceptable fit to the data. The best fitting parameters for a joint RGS1/2 fit are $k T_{\mathrm{BB}}=84.2 \pm 0.4 \mathrm{eV}$, and a column density of $N_{\mathrm{H}}=2.7 \pm 0.310^{20} \mathrm{~cm}^{-2}$. At first sight, one might be tempted to identify coherent features in the post-fit residuals. But careful inspection shows that none of these appear significantly in both spectrometers simultaneously, and so we conclude that the spectrum appears smooth, to within the limits implied by the counting statistics fluctuations in this observation. Based on our experience with similarly smooth continuum spectra in the 
RGS, we roughly estimate that features with an equivalent width larger than approximately one-fifth of the spectral resolution (or $W_{\lambda} \gtrsim 0.01 \AA$ ) should have been detectable.

The spectrum obtained with the PN camera, although of lower spectral resolution than the RGS spectrum, covers a wider band than RGS, and has higher signal to noise per resolution element, and we use it to investigate possible subtle broad-band deviations from the simple black-body shape. A PN spectrum was accumulated using single-pixel events for which a relatively advanced response matrix exists. From the total exposure of $62.5 \mathrm{ks}$ the last $\sim 10 \mathrm{ks}$ and some shorter intervals could not be used for the analysis due to strong background flaring which saturated the data handling system. The total average source count rate is 6.1 counts $\mathrm{s}^{-1}$ (not yet corrected for deadtime). The spectrum was satisfactory fit with an absorbed blackbody model. The best fit parameters are a column density of $N_{\mathrm{H}}=5.8 \pm 0.410^{19} \mathrm{~cm}^{-2}$, and a temperature of $k T_{\mathrm{BB}}=86.0 \pm 0.3 \mathrm{eV}$ with formal $1 \sigma$ errors. The column density is a factor of two lower than derived from the ROSAT PSPC spectrum by Haberl et al. (1997), which is probably caused by calibration uncertainties at the very low energies in both instruments. There is also a formal discrepancy between the column densities measured with RGS and PN, probably due to the fact that RGS is less sensitive to small column densities, and the presence of a small remaining calibration uncertainty around the $\mathrm{O} \mathrm{K}$ edge in RGS. Fixing the column density at the best fitting PN value would slightly increase the RGS blackbody temperature, to $k T_{\mathrm{BB}}=89.2 \pm 0.4 \mathrm{eV}$.

A combined fit of the RGS, EPIC-PN, and ROSAT PSPC spectra is shown in Fig. 2. Both RGS spectra are superimposed, and all spectra have been rebinned such that each spectral bin contains at least 20 counts. The best fit column density and temperature are $N_{\mathrm{H}}=$ $6.0 \pm 0.410^{19} \mathrm{~cm}^{-2}$ and $k T_{\mathrm{BB}}=86.2 \pm 0.3 \mathrm{eV}$, and these values are dominated by the $\mathrm{PN}$ spectrum due to its high statistical quality. The absolute normalizations are still preliminary as accurate exposure and deadtime calculations are not finalized yet. Residuals in the PN fit around $0.28 \mathrm{keV}$ are caused by inaccurate filter transmission data near the carbon K-edge of the thin filter.

\subsection{Neutron star atmospheres}

We also applied neutron star atmosphere models to the RGS and PN data. The X-ray spectra emitted by strongly magnetized atmospheres should exhibit a number of spectral features. First of all, there are electron and proton cyclotron resonances, at energies of $E_{\mathrm{B}_{\mathrm{e}}}=11.6 B_{12} \mathrm{keV}$ and $E_{\mathrm{B}_{\mathrm{p}}}=6.3 B_{12} \mathrm{eV}$, respectively, where $B=B_{12} 10^{12} \mathrm{G}$ is the neutron star surface magnetic field (cf. Pavlov et al. 1995; Zane et al. 2000). Absence of any significant absorption features in the RGS spectra in the 0.35 $1.25 \mathrm{keV}$ range would appear to exclude magnetic fields of $B \simeq(0.3-2.0) 10^{11}$ and $(0.5-2.0) 10^{14}$ G. Similarly, we do not detect a significant cyclotron emission feature,

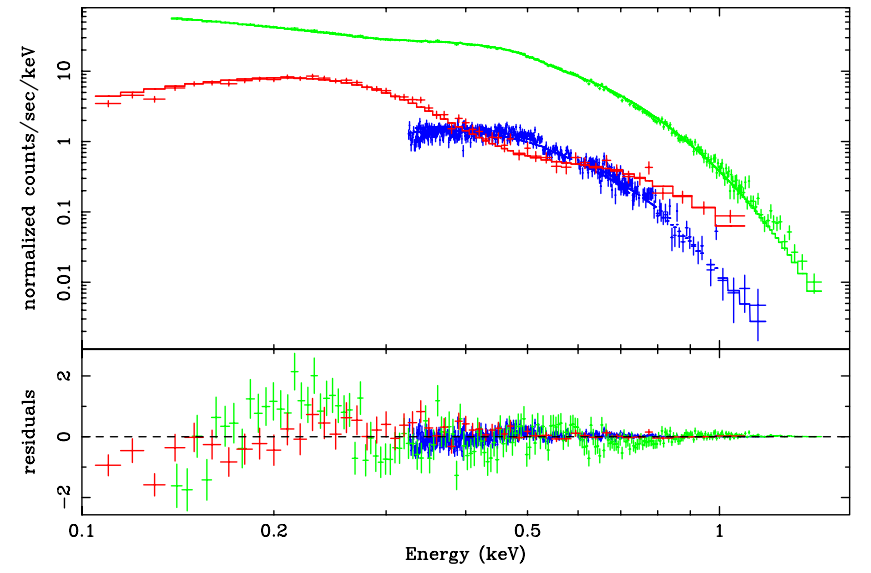

Fig. 2. Combined blackbody fit to the EPIC-PN (green), RGS (blue) and ROSAT PSPC (red) spectra of RX J0720.4-3125

such as has been predicted by Nelson et al. (1993), for a Coulomb heated neutron star atmosphere. We note that these conclusions are relatively robust, because cyclotron features are expected to appear in the spectrum regardless of the surface composition, assuming that the atmosphere is not predominantly neutral (which may be the case for very strong fields).

As far as atomic transitions are concerned, their energies and shapes are of course strongly dependent on the conditions in the atmosphere, and our non-detection of any strong spectral features can therefore only place model-dependent constraints on the stellar parameters. Roughly speaking, there are four possible classes of atmospheric models: those with, and without a strong magnetic field, and those of pure hydrogen composition as opposed to metal-enriched or even pure metal composition. We regard any predictions with respect to either of these parameters as sufficiently uncertain that it makes sense to compare our spectrum to predictions for all four cases.

We start with pure hydrogen atmospheres. At temperatures below $10^{6} \mathrm{~K}$, the spectra of magnetized hydrogen atmospheres have pronounced features in the soft $\mathrm{X}$-ray range due to bound-bound transitions in the hydrogen atom (Pavlov \& Zavlin 2001). The energy of the strongest transition is estimated by the formula $E \simeq$ $0.052+0.009 \ln B_{12}+0.006 B_{12} \mathrm{keV}$. This excludes magnetic fields of $B>410^{13} \mathrm{G}$. On the other hand, fits with hydrogen atmosphere models for $B<10^{10} \mathrm{G}$ and $B=10^{11}-10^{13} \mathrm{G}$ (which have no discrete structure in our band) require a surface temperature of $\sim 310^{5} \mathrm{~K}$, significantly lower than that given by the blackbody fit. However, the shapes of these very low or high B-field atmospheric spectra are sufficiently similar to blackbodies (of very different effective temperature, however) that we cannot distinguish between blackbody and hydrogen atmosphere models on statistical grounds.

Low-field models with significant metal abundance (either solar abundances, or pure iron) do not fit the data because of the strong atomic absorption features in the spectra (Rajagopal \& Romani 1996; Zavlin et al. 1996). 


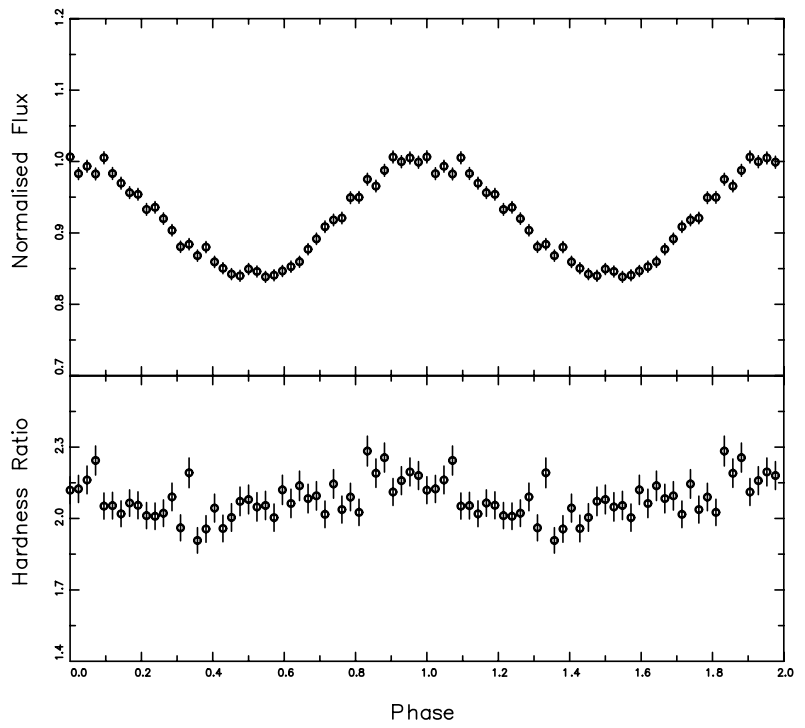

Fig. 3. The total flux (top) from EPIC-PN phase folded on the $8.391 \mathrm{~s}$ period of Haberl et al. (1997) with the (0.1 to 0.4$) /(0.4$ to 0.8 ) $\mathrm{keV}$ hardness ratio (bottom). The flux is normalised at phase 0.0

The case of strongly magnetized metal-enriched atmospheres is more difficult to assess due to the relative scarcity of relevant calculations. The strongly magnetized pure Fe spectra presented by Rajagopal et al. (1997) show significant absorption structure in the RGS band (for sufficiently high $T_{\text {eff }}$ that the radiation is detectable), but the structure may be subtle enough (dense, and small contrast) that it may not be easy to detect in data of finite resolution and statistical quality. We regard the absence of strong features in our spectrum as inconclusive in this respect.

\section{The X-ray pulsation}

We have accumulated counts from the EPIC-PN detector over the energy ranges $0.1-0.4,0.4-0.8$ and $0.8-1.2 \mathrm{keV}$ and phase-averaged them over 42 phase intervals on the $8.391 \mathrm{~s}$ ephemeris of Haberl et al. (1997). We show the intensity curve in the $0.1-1.2 \mathrm{keV}$ energy band together with the hardness ratio (soft/medium) in Fig. 3. Because of its poorer time resolution we have not used the EPICMOS data.

The general shape of the phased intensity curve is similar to that found by Haberl et al. (1997) although the uncertainties are smaller. It appears approximately symmetrical and sinusoidal; the amplitude is $\sim 15 \%$. Very interestingly, the hardness ratio is also seen to vary, in the sense that it is softest around flux maximum, but the amplitude is smaller $(\sim 10 \%)$. The phasing of the hardness ratio curve is slightly but significantly earlier than the intensity curve. A cross-correlation of the two curves indicates that the phase difference between maximum intensity and maximum hardness is $\Delta \phi=-0.048$.

Because of the uncertainty in absolute timing for our data, we are currently unable to refine the ephemeris to the level required to discern any changes in the period of the X-ray pulsation with respect to the period as measured with ROSAT. A Fourier analysis of the data using an arbitrary starting time yields only one significant period, at $8.3912 \mathrm{~s}$, consistent with that reported by Haberl et al. (1997).

The high $\mathrm{S} / \mathrm{N}$ ratio of the X-ray pulsation in these data provides the opportunity for a more detailed modeling of the angular and spatial distribution of the radiation than was possible from the ROSAT data, and this analysis will be presented in a companion paper (Cropper et al. 2001).

\section{Conclusions}

We present the atmospheric spectrum of the isolated neutron star RX J0720.4-3125, as measured with the RGS and EPIC-PN instruments on XMM-Newton. We find that the spectral shape is smooth, without evidence for strong spectral features. The absence of electron or proton cyclotron resonances in the RGS band would appear to exclude average surface magnetic field strengths of $B \simeq(0.3-2.0) 10^{11}$ and $(0.5-2.0) 10^{14} \mathrm{G}$. The implications of the apparent absence of atomic transitions are strongly model dependent, and we can therefore not rule out either a pure hydrogen atmosphere or a metal-rich atmosphere, if the magnetic field strength satisfies certain restrictions.

Acknowledgements. The Columbia group is supported by the US National Aeronautics and Space Administration. We gratefully acknowledge comments from the referee, George Pavlov.

\section{References}

Cropper, M., Zane, S., Ramsay, G., Haberl, F., \& Motch, C. 2001, A\&A, 365, L302

Greenstein, G., \& Hartke, G. J. 1983, ApJ, 271, 283

Haberl, F., Motch, C., Buckley, D. A. H., Zickgraf, F.-J., \& Pietsch, W. 1997, A\&A, 326, 662

den Herder, J. W., Brinkman, A. C., Kahn, S. M., et al. 2001, A\&A, 365, L7

Jansen, F., Lumb, D., Altieri, B., et al. 2001, A\&A, 365, L1

Kulkarni, S. R., van Kerkwijk, M. H. 1998, ApJ, 507, L49

Motch, C., \& Haberl, F. 1998, A\&A, 333, L59

Nelson, R. W., Salpeter, E. E., \& Wasserman, I. 1993, ApJ, 418,874

Pavlov, G. G., Shibanov, Yu. A., Zavlin, V. E., \& Meyer, R. D. 1995, in The Lives of the Neutron Stars, ed. M. A. Alpar, Ü. Kiziloğlu, \& J. van Paradijs (Dordrecht: Kluwer), 71

Pavlov, G. G., \& Zavlin, V. E. 2001, in preparation

Rajagopal, M., \& Romani, R. W. 1996, ApJ, 461, 327

Rajagopal, M., Romani, R. W., \& Miller, M. C. 1997, ApJ, 479,347

Strüder, L., Briel, U., Dennerl, K., et al. 2001, A\&A, 365, L18

Turner, M. J. L., Abbey, A., Arnaud, M., et al. 2001, A\&A, 365, L27

Zane, S., Turolla, R., \& Treves, A. 2000, ApJ, 537, 387

Zavlin, V. E., Pavlov, G. G., \& Shibanov, Yu. A. 1996, A\&A, 315,141 